\title{
Análisis regional de las islas de calor urbano en la Argentina
}

\author{
Paula Casadei ${ }^{1, \otimes}$; María Semmartin ${ }^{2,3}$ \& Martín F. Garbulsky ${ }^{1,3}$ \\ ${ }^{1}$ Cátedra de Forrajicultura, Facultad de Agronomía, Universidad de Buenos Aires. ${ }^{2}$ Cátedra de Ecología, Facultad de \\ Agronomía, Universidad de Buenos Aires. ${ }^{3}$ IFEVA - Universidad de Buenos Aires/CONICET. Ciudad Autónoma de Buenos \\ Aires, Argentina.
}

\begin{abstract}
RESUMEN. La urbanización es una de las formas más extremas de cambios en el uso de la tierra y tiene impactos sobre el clima, el agua y la biodiversidad en grandes áreas de todo el planeta. En este trabajo se cuantificaron las islas de calor urbano (ICU) y se relacionaron con características de las ciudades y su entorno a lo largo de gradientes ambientales de la Argentina. Analizamos 55 de las ciudades más grandes del país, ubicadas en 10 ecorregiones, mediante datos satelitales (MODIS) de temperatura de la superficie terrestre, albedo e índices de vegetación (NDVI), y de variables climáticas. La ICU diurna promedio anual de las ciudades argentinas para 2011-2015 fue $0.36 \pm 1.99{ }^{\circ} \mathrm{C}$ y la nocturna $1.68 \pm 0.61{ }^{\circ} \mathrm{C}$. Los principales controles de la intensidad de la ICU fueron el NDVI rural, la precipitación media y la temperatura media durante el día y, en menor medida, la diferencia del albedo durante la noche. Durante el día, algunas ciudades se comportaron como islas de frío urbano (IFU) asociadas a climas áridos o a contextos agrícolas, mientras que durante la noche, todas las ciudades se comportaron como ICU. El efecto atemperador de la vegetación urbana se identificó a partir de la diferencia de NDVI urbano y rural, pero no se observó una relación directa negativa de ICU con NDVI urbano. Los resultados de este trabajo proveen nuevos conocimientos sobre los controles de las ICU y permitiría generar estrategias de desarrollo urbano para mitigar los efectos de la urbanización y mejorar la calidad de vida de la población urbana.
\end{abstract}

[Palabras clave: ecología urbana, temperatura de superficie, NDVI, albedo, teledetección]

\begin{abstract}
Aвstract. Regional analysis of urban heat islands in Argentina. Urbanization is one of the most extreme land-use changes and has impacts on climate, water and biodiversity in large areas throughout the planet. In this paper, we quantified the urban heat islands (ICU, acronym in Spanish) and their relation to biophysical parameters of the surface of the earth along the main environmental gradients of Argentina. We analyzed 55 of the largest cities in the country, located in 10 ecoregions, using satellite data (MODIS) of land surface temperature, albedo and vegetation indexes (NDVI) and climatic variables. The average annual ICU in the cities for $2011-2015$ was $0.36 \pm 1.99^{\circ} \mathrm{C}$ for the day and $1.68 \pm 0.61{ }^{\circ} \mathrm{C}$ for the night. The main drivers of the ICU during the day were rural NDVI, mean precipitation and temperature. At night, the main driver was the difference between urban and rural blue albedo. During the day, some cities behaved like urban cold islands (IFU, acronym in Spanish) associated with arid climates or agricultural contexts, while at night, all cities behaved like ICU. The cooling effect of urban vegetation was identified from the difference between urban and rural NDVI, but no direct negative relationship between ICU and urban NDVI was observed. These results provide new insights on the drivers of the ICU and facilitate the generation of urban development strategies to mitigate the effects of urbanization and to improve the quality of life of urban populations.
\end{abstract}

[Keywords: urban ecology, surface temperature, NDVI, albedo, remote sensing]

\section{INTRODUCCIÓN}

Las áreas urbanizadas ocupan sólo 2\% de la superficie terrestre, aunque en ellas reside más de la mitad de la población mundial (Bonet et al. 2011). Los procesos de urbanización se han acelerado, por lo que la población urbana hoy supera por primera vez a la población rural (Naciones Unidas 2018). Se estima que para el año 2030, el 60\% de la población mundial vivirá en zonas urbanas (Mills 2007) y la cobertura global urbana pasará de 605000 $\mathrm{km}^{2}$ en 2000 a más de $1900000 \mathrm{~km}^{2}$ en 2050 (Angel et al. 2011).

La urbanización representa un cambio extremo en el uso de la tierra (Groffman et

\section{Editor asociado: Germán Baldi}

al. 2014). La remoción de cobertura vegetal y su reemplazo por nuevas superficies -impermeables en su mayoría - alteran la estructura y funcionamiento de los ecosistemas y pueden modificar el clima, la biodiversidad y los ciclos del agua, del carbono y de los nutrientes (Grimm et al. 2008). Uno de los impactos de la urbanización es el aumento de la temperatura respecto a su entorno no urbanizado, denominado isla de calor urbano (ICU) (Oke 1976). Durante el día, las propiedades térmicas de los materiales de construcción, el bloqueo del viento por los edificios (Taheri Shahraiyni et al. 2016) y cambios en las tasas de intercambio de calor (Imhoff et al. 2010; Paoloni 2012) modifican la 
energía disponible en el área urbana, calor que es liberado durante la noche principalmente. El suelo impermeabilizado modifica propiedades de la superficie que contribuyen a la ICU como el albedo y la emisividad, lo que se puede observar a partir de imágenes satelitales (Jin 2005).

El impacto de la urbanización depende del lugar de emplazamiento de la ciudad, el grado de alteración del ambiente original, la extensión y distribución de las superficies afectadas (Imhoff et al. 2010). La intensidad de ICU se ha correlacionado con la extensión y la densidad de la urbanización (Tan and Li 2015; Zhou et al. 2017). La intensidad de la transformación producida por la urbanización y el planeamiento de espacios verdes también influye en la ICU al contribuir al enfriamiento del aire. La vegetación urbana tiene un rol fundamental en el control de la temperatura durante el día (Buyantuyev and Wu 2010; Gioia et al. 2014), principalmente a partir de evapotranspiración y disipación de calor en la atmósfera y la provisión de sombra a las superficies (Feyisa et al. 2014; Lazzarini et al. 2013). Sin embargo, el análisis del contexto ecológico (i.e., factores biofísicos como clima o cobertura) permite identificar otros controles a escala regional. Por ejemplo, las áreas urbanas son en promedio más cálidas que las no urbanas, salvo aquellas áreas urbanas ubicadas en climas áridos y semiáridos (Imhoff et al. 2010; Taheri Shahraiyni et al. 2016). Las escalas temporales también determinan la intensidad de la ICU. A nivel diario, zonas templadas pueden tener isla de calor durante el día (Taheri Shahraiyni et al. 2016), aunque las mayores diferencias ocurren luego del atardecer (Roth et al. 1989). Si se analiza estacionalmente, en zonas templadas o subtropicales la intensidad es mayor durante estaciones cálidas (Jenerette et al. 2011), mientras que ciudades tropicales exhiben una intensidad mayor en temporadas secas o frías (Roth 2007).

Las ICU controlan el incremento del consumo energético y la contaminación ambiental (Correa et al. 2006) y tienen impactos sobre la productividad de la vegetación de espacios urbanos (Walker et al. 2015), el establecimiento de especies exóticas (McKinney 2006) e, incluso, sobre la salud humana (Wong et al. 2013). Por lo tanto, describir los impactos de las islas de calor puede generar cimientos para otros estudios relacionados con la ecología urbana y el ordenamiento territorial. En la Argentina existen algunos análisis a escala local del impacto de la urbanización sobre el aumento de la temperatura urbana (e.g., para Santa Teresita [Verón 2010], el Gran San Miguel de Tucumán [Paolini 2012] y Córdoba [Maristany et al. 2008; de la Casa and Nasello 2014]). Hasta el momento no se realizaron estudios a escala regional que integren los resultados de diferentes áreas urbanas y que faciliten la comprensión de la importancia del contexto de la urbanización.

Los sensores remotos permiten estudiar el comportamiento de la superficie terrestre en el espacio y en el tiempo (Garbulsky et al. 2011, 2014), y acceder a lugares de interés cuando los recursos para el relevamiento a campo son escasos. El fenómeno ICU se puede derivar a partir de mediciones de la temperatura de la superficie terrestre (TST). La TST suele ser más alta y variable que la temperatura del aire, pero se puede relacionar más fácilmente con las condiciones de la superficie (Streutker 2002).

En este trabajo combinamos información satelital para caracterizar a nivel regional el impacto de la urbanización sobre la temperatura de superficie en la Argentina, abarcando la heterogeneidad ambiental del país. Los objetivos son describir y cuantificar las islas de calor urbano en relación con características de las ciudades y su entorno a lo largo de gradientes ambientales, y analizar la influencia de la vegetación urbana en la intensidad de las islas de calor.

\section{Materiales y Métodos}

Se estudiaron 55 ciudades de la Argentina (Figura 1; Tabla S1, Material Suplementario), elegidas entre 100 ciudades con más de 50000 habitantes (INDEC 2010) ubicadas en un amplio gradiente de precipitación anual (de 150 a $1900 \mathrm{~mm} / \mathrm{año}$ ) y temperatura media (de 13 a $25{ }^{\circ} \mathrm{C}$ ) (promedio de 15 años; Global Climate Monitor de la Universidad de Sevilla; www.globalclimatemonitor.org) y en 10 ecorregiones (Olson et al. 2001). De las 100 ciudades se excluyeron las urbanizaciones pertenecientes a áreas metropolitanas de grandes ciudades y aquellas ubicadas en la Estepa Patagónica o en los Bosques AndinoPatagónicos debido a la dificultad de ubicar áreas urbanizadas/no urbanizadas en situaciones ambientales similares.

Se delimitaron las áreas urbanas a partir de una clasificación de uso de la tierra basada en información satelital (Friedl et al. 2010). 


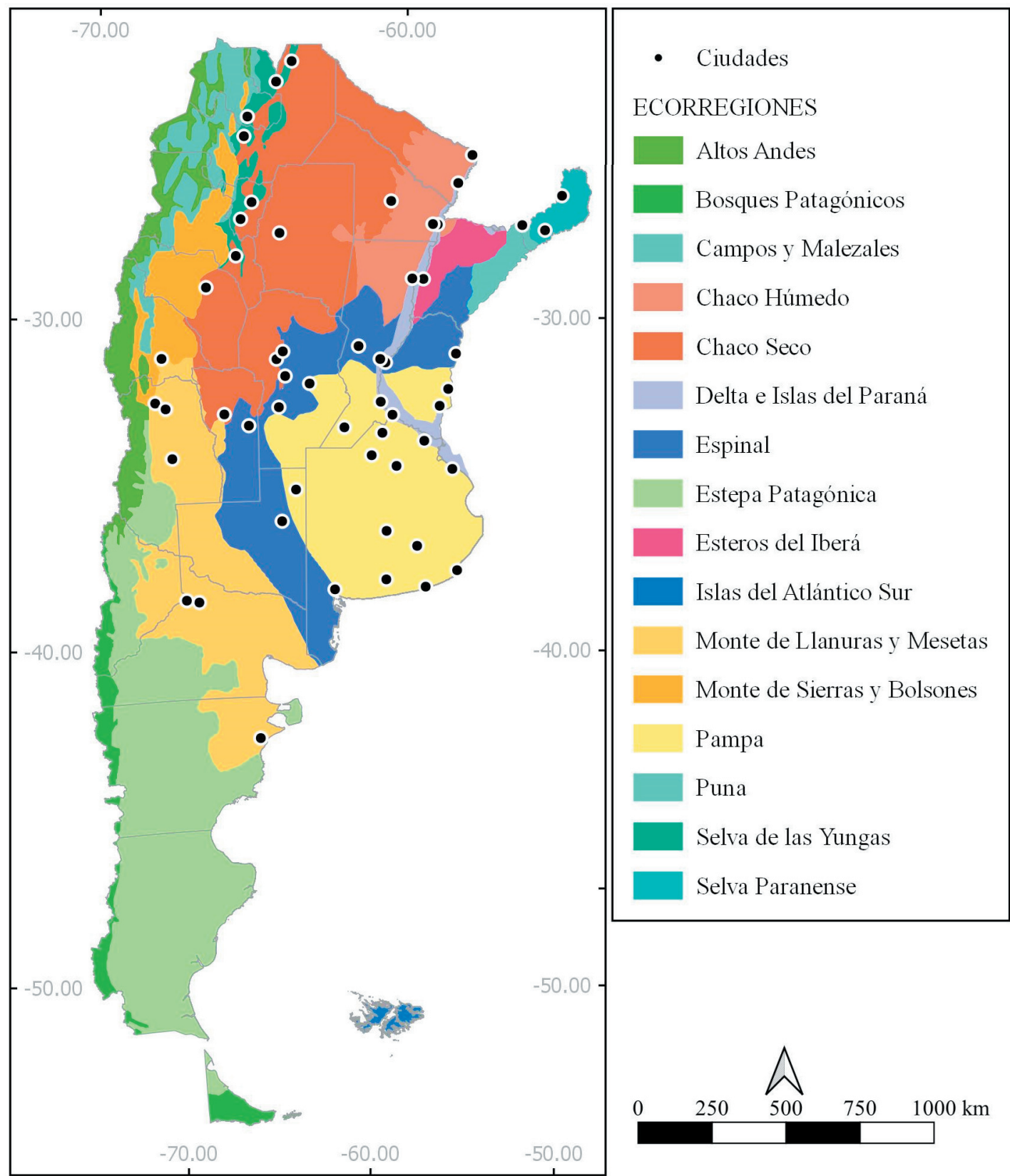

Figura 1. Ubicación de las 55 ciudades analizadas en el contexto de las ecorregiones (Olson et al. 2001). Figure 1. Location of 55 cities analyzed in the ecoregions context (Olson et al. 2001).

La definición de áreas urbanas y no urbanas se realizó mediante fotointerpretación de las imágenes satelitales de alta resolución (Google Earth), junto a una base de datos de planta urbana (IGN 2019). El punto urbano se ubicó sobre la zona más densamente construida, considerando la proporción de espacio vegetado (público y privado) y la densidad y altura de los edificios. Esta aproximación permitió establecer el área con mayor superficie impermeable (indicador de uso urbano) para obtener la temperatura de superficie correspondiente (TSTu). La extensión de la superficie urbana fue calculada en QGIS para cada localidad considerando su área metropolitana. Se calculó la isla de 
calor urbano (ICU) como la diferencia de temperatura entre zonas urbanas y no urbanas según la siguiente ecuación:

\section{ICU = TSTu - TSTnu}

donde TSTu: temperatura de superficie terrestre urbana; TSTnu: temperatura de superficie terrestre no urbana

Para cuantificar las ICU se calculó la diferencia de temperaturas por fecha para el período 2011-2015, cuyos promedios permitieron describir cuantitativamente las ICU según diferentes escalas temporales (e.g., mensual, estacional, anual).

La temperatura no urbana (TSTnu) fue definida por cuatro puntos en zonas rurales cercanas a la ciudad, que integran la heterogeneidad de usos del suelo (Figura 2). Se realizó un análisis preliminar que permitió caracterizar el gradiente horizontal de temperatura a lo largo de un área urbana. De esta manera, se buscó conocer si existe efecto deslocalizado de la isla de calor, es decir, hasta qué distancia fuera de la ciudad se percibe su influencia sobre la temperatura (Material Suplementario). A los 10 kilómetros del punto central no se detectó influencia de la urbanización, ya que se mantenía constante el valor de ICU. La isla de calor urbano disminuyó gradualmente con el aumento de la distancia al centro urbano de manera similar a lo encontrado en la bibliografía (Han and Xu 2013).

Se extrajo y promedió la información de temperatura de los cuatro puntos a $10 \mathrm{~km}$ de distancia desde el centro de cada ciudad (Figura 2). Se evitaron aquellos puntos no urbanos que caen sobre otras áreas urbanas, en elevaciones topográficas con diferencias mayores a $50 \mathrm{~m}$ (o el 10\%) de la elevación media del centro urbano, o sobre cuerpos de agua (Imhoff et al. 2010). Para la diferencia de altitud se utilizó el modelo de elevación digital SRTM Digital Elevation Data (Farr et al. 2007). Al considerar que la isla de calor es indicadora del impacto de la ciudad sobre el microclima se decidió elegir puntos sobre áreas no urbanas a)

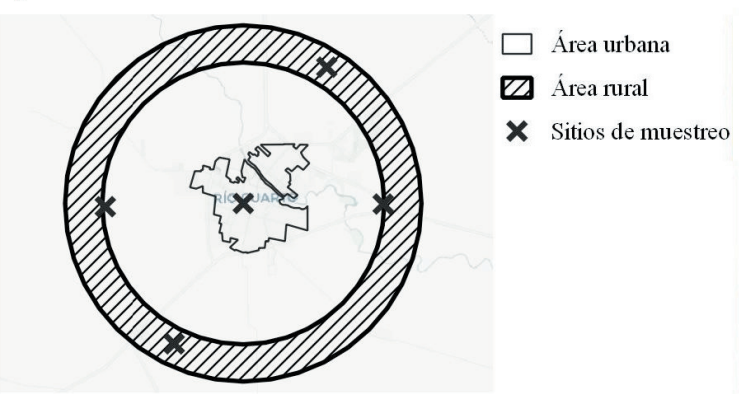

c)

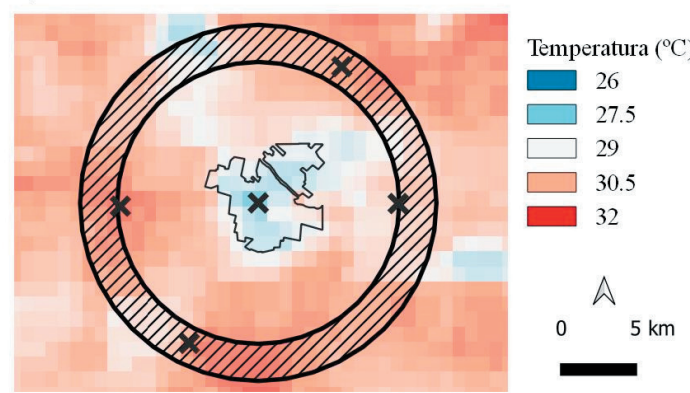

b)

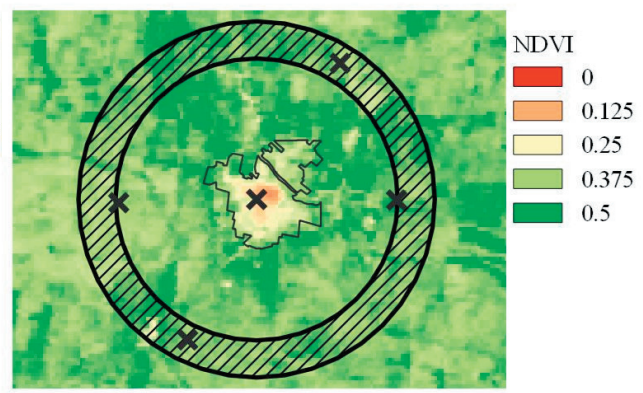

d)

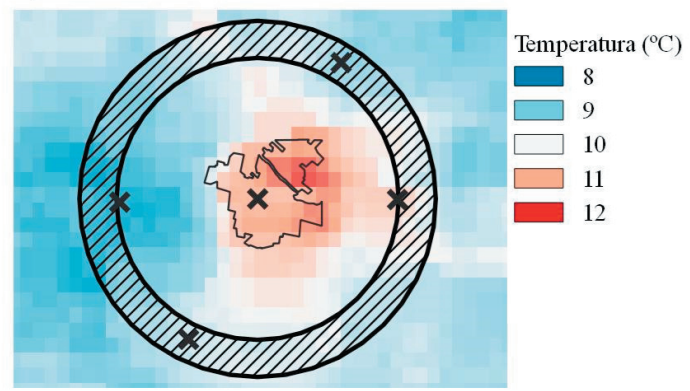

Figura 2. Información satelital y ubicación de los puntos de muestreo para la ciudad de Río Cuarto (Córdoba). a) Área de muestreo rural (área con líneas) a $10 \mathrm{~km}$ desde el punto urbano, ubicado dentro del área urbana (sin relleno). Las cruces indican píxeles elegidos para extraer datos MODIS. b) NDVI promedio 2011-2015, con resolución 250 m. c) Temperatura de superficie diurna promedio 2011-2015, con resolución $1 \mathrm{~km}$. d) Temperatura de superficie nocturna promedio 2011-2015, con resolución $1 \mathrm{~km}$.

Figure 2. Satellite information and location of sampling points for Río Cuarto (Córdoba). a) Rural sampling area (area with lines) considering $10 \mathrm{~km}$ from the urban point, located within the urban area (without landfill). The crosses indicate the pixels chosen to extract data from MODIS. b) Average NDVI 2011-2015, with resolution 250 m. c) Average daytime surface temperature 2011-2015 at $1 \mathrm{~km}$ resolution. d) Average nighttime surface temperature 2011-2015, at $1 \mathrm{~km}$ resolution. 
similares al lugar de establecimiento de la ciudad. Por ejemplo, para Rosario se evitó el Delta y en las ciudades ubicadas en valles se muestreó sólo en sitios no urbanos dentro del valle.

Las ciudades se describieron en función de variables sociodemográficas (habitantes, superficie) y biofísicas agrupadas bajo la categoría de ecorregión y de otras variables individuales. Se incluyeron precipitación anual media, temperatura anual media, vegetación (urbana y no urbana) y albedo (urbano y no urbano), derivadas de sensores remotos.

Se utilizó información satelital MODIS (del inglés Moderate Resolution Imaging Spectroradiometer) para obtener datos de TST, derivada de emisividad térmica, albedo e índice de vegetación de diferencia normalizada (NDVI por sus siglas en inglés) del período 2011-2015. Los sensores MODIS montados sobre los satélites TERRA y AQUA, de órbita polar, realizan dos pasajes diarios (diurno y nocturno). Se utilizaron los productos de temperatura de superficie (MYD11), NDVI (a partir de MOD09) y albedo (MCD43), obtenidos de MODIS Land Product Subsets-NASA (http://daac.ornl.gov/MODIS/ modis.shtml) o través de Google Earth Engine (code.earthengine.google.com). Estos datos satelitales permiten analizar la variabilidad temporal con una resolución espacial de 250 a $1000 \mathrm{~m}$.

La temperatura de superficie (MYD11) (Wan et al. 2015) fue utilizada para caracterizar el gradiente horizontal de temperatura a lo largo del área urbana. Este producto provee un valor de temperatura diario $\left({ }^{\circ} \mathrm{K}\right)$ entre las 13:00 y las 15:00 h, y entre las 00:00 y las 2:00 h hora local, respectivamente, cercanos a los momentos de máximos y mínimos de temperatura diaria. El análisis temporal de TST 2011-2015 se realizó con el producto MYD11A2, cada 8 días por píxel de $1 \mathrm{~km}^{2}$, que promedia los valores diarios de temperatura registrados en días sin nubes (Wan 2006). Se filtraron los datos con errores mayores a $2^{\circ} \mathrm{K}$ utilizando la banda Quality Control (Zhou et al. 2017).

Se calculó el NDVI, un estimador del estado de la vegetación y la productividad primaria (Pettorelli et al. 2005), como NDVI=(IR$\mathrm{R}) /(\mathrm{IR}+\mathrm{R})$, donde IR es la reflectancia en el infrarrojo (banda 2 de MODIS 841-876 nm) y $\mathrm{R}$ es la reflectancia en el rojo (banda 1 , $620-670 \mathrm{~nm}$ ) a partir de reflectancia de la superficie (MOD09Q1) con resolución de 250 m y cada 16 días (lpdaac.usgs.gov/products/ mod09q1v006). Se calculó la diferencia entre cobertura urbana y el promedio no urbano. Se caracterizó el píxel urbano según su NDVI bajo el supuesto que un píxel con mayor superficie impermeable tiene un menor NDVI. El NDVI de las ciudades integra la reflectancia de todas las superficies incluidas, por lo que se asume que la diferencia entre ciudades está determinada exclusivamente por el funcionamiento de la vegetación. Con esta metodología se consideran los espacios verdes públicos y privados y el arbolado urbano. Para que este supuesto sea más robusto, se obtuvieron valores de albedo (producto MCD43) de estas mismas superficies que integra a las construcciones urbanas.

El albedo fue obtenido del producto MCD43B3, generado a partir de la información combinada de TERRA y AQUA (lpdaac.usgs.gov/products/mcd43a4v006), con resolución espacial de $1 \mathrm{~km}$ y temporal de 8 días. El albedo es la fracción de la radiación solar reflejada por la Tierra respecto a la radiación incidente, juega un rol clave en el balance de radiación de la tierra e influye en la temperatura anual media (Porteous 2013). Es un indicador de la absorción de radiación y las propiedades térmicas de la superficie urbana. Durante la noche, su emisión contribuye a mantener elevada la temperatura en las ciudades. Se calculó el albedo azul como el promedio entre el albedo blanco y el negro, provistos por MODIS. Se utilizó la diferencia entre el albedo azul urbano y el no urbano como indicador del almacenamiento de energía en la superficie (Peng et al. 2012).

Las variables que caracterizaron a la ciudad y su entorno se remuestrearon de manera que coincida la resolución espacial con la resolución de la TST. Las relaciones entre las islas de calor y los factores ambientales se analizaron principalmente con el software $\mathrm{R}$ (www.r-project.org).

\section{Resultados}

\section{Distribución y caracterización de islas de calor en la Argentina}

Las diferencias entre la temperatura superficial urbana y no urbana en las 55 ciudades de la Argentina analizadas mostraron la existencia extendida del fenómeno de isla de calor. El promedio anual diurno para todas 

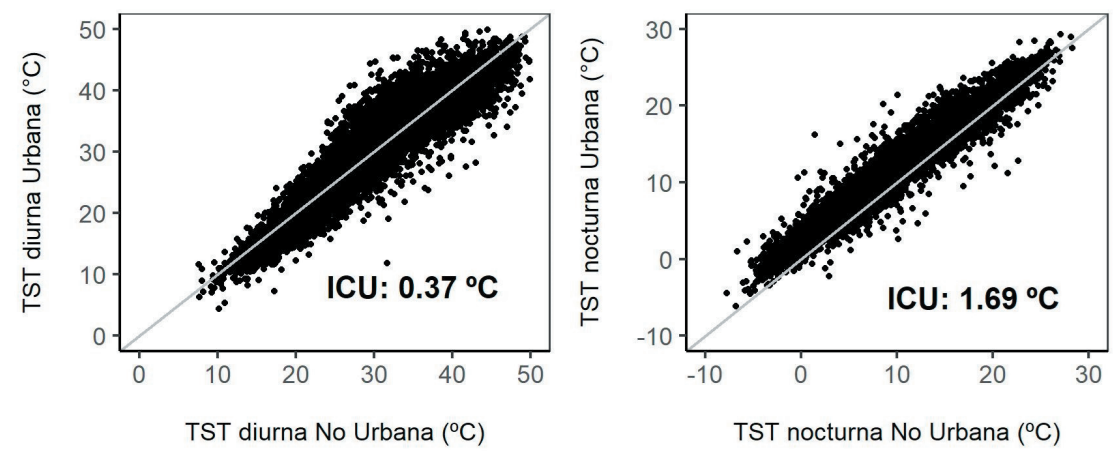

Figura 3. Relación entre la temperatura de superficie urbana y el promedio de los píxeles no urbanos $(n=4)$, durante (a) el día y (b) la noche (2011-2015), para todas las fechas con buena calidad ( $n=12650)$. La línea gris indica la relación 1:1. La ICU promedio diurno (a) fue $0.37^{\circ} \mathrm{C}$ y la nocturna (b) fue $1.69^{\circ} \mathrm{C}$.

Figure 3. Relationship between urban and mean for non-urban surface temperature pixels $(n=4)$ during (a) daytime and (b) nighttime (2011-2015), for all dates with good quality $(n=12650)$. The gray line indicates the 1:1 ratio. The ICU average diurnal (a) was $0.37^{\circ} \mathrm{C}$, and nocturnal (b) was $1.69^{\circ} \mathrm{C}$.

las fechas con buena calidad fue $0.37^{\circ} \mathrm{C}$ y el nocturno $1.69^{\circ} \mathrm{C}(n=12650)$ (Figura 3$)$. El $43.5 \%$ de los datos diurnos tuvieron TST urbanas mayores a las no urbanas, lo que indica la presencia de la ICU, que se acentuó en el rango de $25-35^{\circ} \mathrm{C}$ de TST no urbanas (Figura 3). Durante la noche, el porcentaje ascendió a $85.6 \%$ y se mantuvo constante a lo largo de todo el gradiente de temperatura no urbana (Figura 3).

El promedio de las ICU (Figura 4) presentó diferencias entre ciudades (valor $P<0.05$ ). Las diferencias diurnas fueron siempre positivas en el norte del país; sin embargo, desde Córdoba hacia el sur existieron situaciones contrastantes. Los extremos están caracterizados por Mendoza $\left(-4.2 \pm 0.44{ }^{\circ} \mathrm{C}\right)$ y Oberá $\left(4.3 \pm 0.42^{\circ} \mathrm{C}\right)$, mientras que San Martín es la ciudad con menores diferencias $(-0.02 \pm 0.20$ $\left.{ }^{\circ} \mathrm{C}\right)$. Estos resultados demuestran la existencia de islas de frío urbano (IFU), presente en $40 \%$ de las ciudades (Figura 4). Durante la noche, la ICU fue similar en todas las ciudades (Figura 4). Orán fue la ciudad de menor ICU nocturna anual promedio $\left(0.36 \pm 0.22^{\circ} \mathrm{C}\right)$, y Villa María, la de mayor $\left(3.1 \pm 0.45^{\circ} \mathrm{C}\right)$.

\section{Posibles controles de la ICU}

Las ICU diurnas correlacionaron de forma positiva con la precipitación anual media y la temperatura anual media (Figura $5 \mathrm{a} \mathrm{y} \mathrm{b),} \mathrm{pero}$ no se encontraron relaciones significativas para las ICU nocturnas (Tabla S2). Las ICU diurnas se correlacionaron positivamente con la diferencia de NDVI no urbano-urbano $\left(R^{2}=0.19\right)$ (Figura 6a). Mayores diferencias de NDVI se correlacionaron con mayores cambios en la TST diurna en el área urbana con respecto al entorno (pero no durante la noche) (Figura
$6 b)$, independientemente de su ubicación geográfica. Al desagregar la diferencia se encontró una relación positiva entre la ICU diurna y el NDVI no urbano promedio anual $\left(R^{2}=0.51 ; P<0.001\right)$, representado por valores entre 0.26 a 0.78 (Trelew y Eldorado, respectivamente). Ciudades ubicadas en zonas con NDVI mayor a 0.5 se caracterizaron generalmente como ICU. En cambio, aquellas ciudades cuyo NDVI no urbano fue menor a 0.5 se comportaron como islas de frío (Figura $\mathrm{S} 2)$.

La vegetación urbana se caracterizó por tener un gradiente de NDVI medio anual urbano que varió entre 0.10 a 0.51 (Tucumán y Eldorado, respectivamente) y se relacionó de forma lineal y positivamente con la ICU durante el día, a nivel regional $\left(R^{2}=0.21 ; P<0.001\right)$. Durante la noche, la relación fue negativa y con menor ajuste $\left(R^{2}=0.08 ; P<0.05\right)$ (Tabla S2).

El albedo azul urbano varió entre 0.127 y 0.190 , con una media de 0.159 , diferente significativamente $(P<0.05)$ a zonas no urbanas que registraron valores entre 0.120 y 0.175 con media en 0.152 . Se encontró una correlación negativa entre la ICU nocturna y la diferencia de albedo entre áreas urbanas y no urbanas $\left(R^{2}=0.1 ; P<0.05\right)$ (Figura 7), mientras que durante el día no se encontró relación. Otras características como la extensión urbana o la cantidad de habitantes no fueron controles importantes de la ICU diurna ni nocturna (Tabla S2).

Las ciudades ubicadas en sitios con mayor temperatura, precipitación o NDVI mostraron ICU más intensas (Figura S4) mientras que las ciudades en el extremo más seco y menos vegetado del gradiente (Monte o Espinal) se 
a)
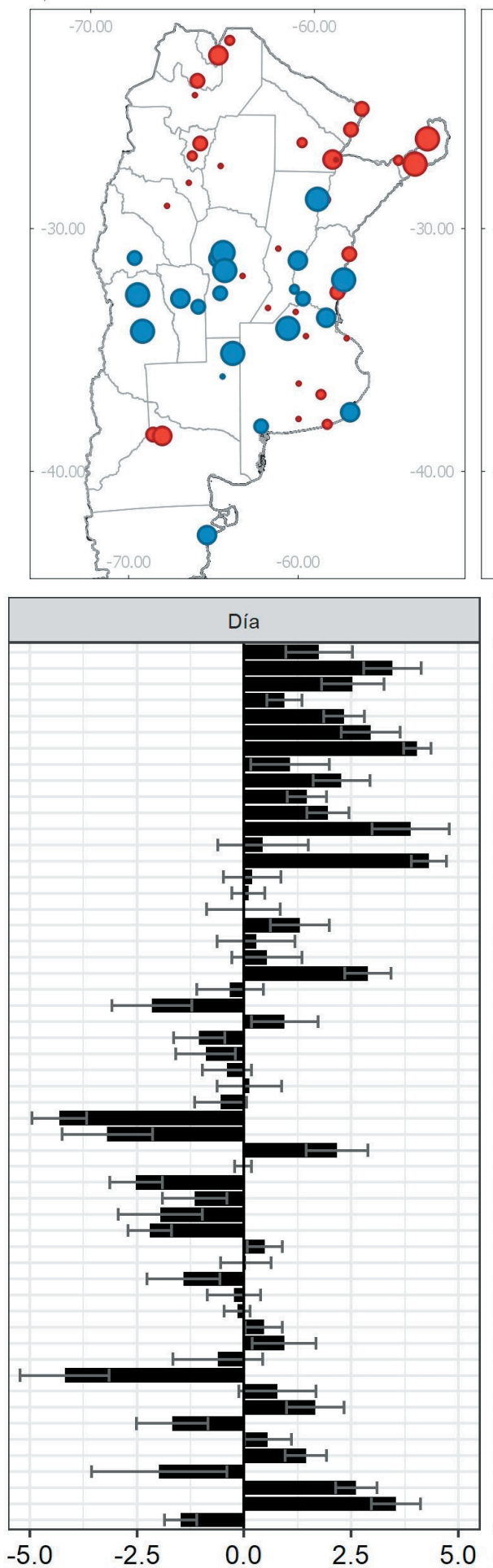

b)

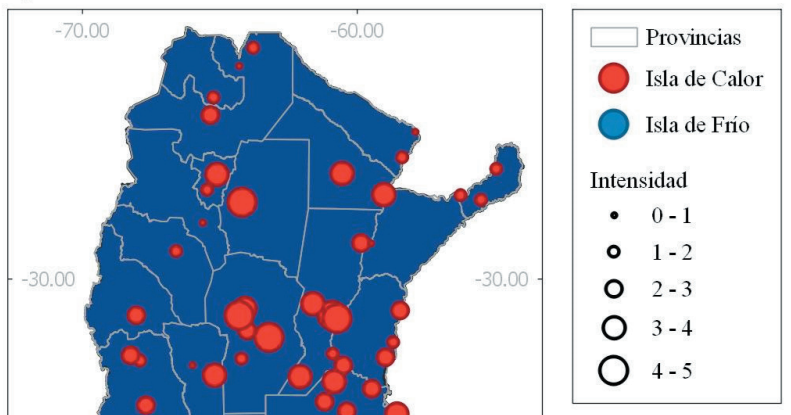

Isla de Calor $\left({ }^{\circ} \mathrm{C}\right)$

Figura 4. Diferencias promedio en la temperatura superficial (a) diurna y (b) nocturna (panel superior) para 55 ciudades de la Argentina, en comparación con sus entornos no urbanizados. Las barras de dispersión (panel inferior) representan el desvío estándar para 2011-2015. La ICU media diurna es $0.36^{\circ} \mathrm{C}$ y la nocturna es $1.68^{\circ} \mathrm{C}$.

Figure 4. Average differences in the surface temperature (a) daytime and (b) nighttime (upper panel) for 55 cities in Argentina, compared to their non-urban surroundings. The scatter bars (lower panel) represent the standard deviation for 2011-2015. Mean diurnal ICU is $0.36^{\circ} \mathrm{C}$ and $1.68{ }^{\circ} \mathrm{C}$ at night. 

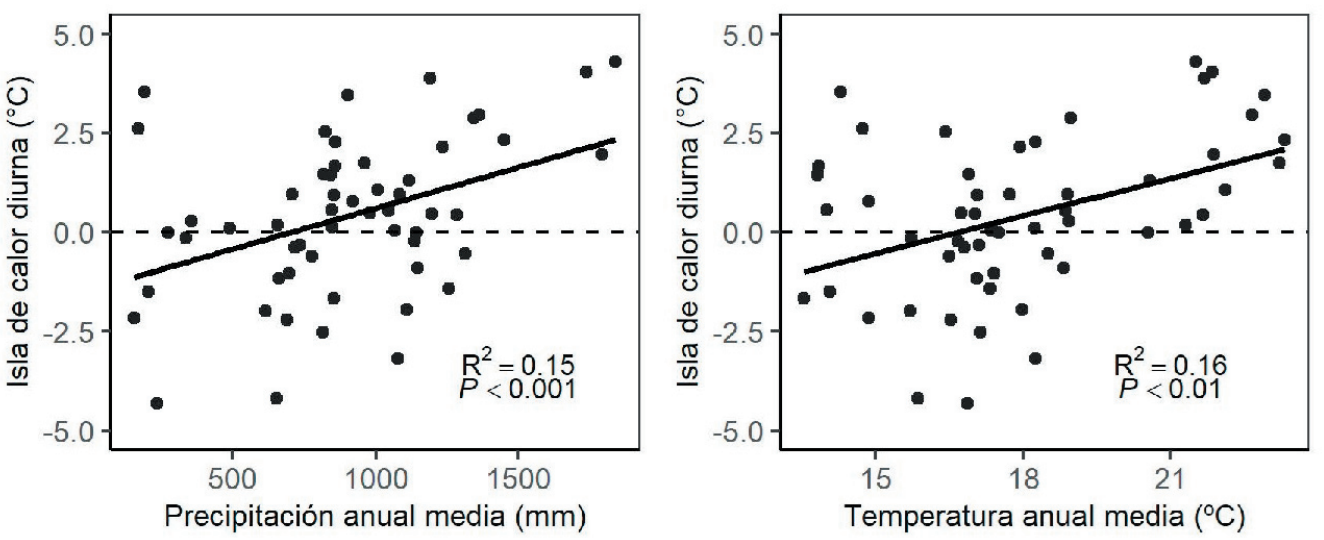

Figura 5. Islas de calor diurna de las 55 ciudades en función de (a) la temperatura anual media, (b) la precipitación anual media.

Figure 5. Daytime heat islands for the 55 cities in relation to (a) the average annual temperature, (b) the mean annual precipitation.
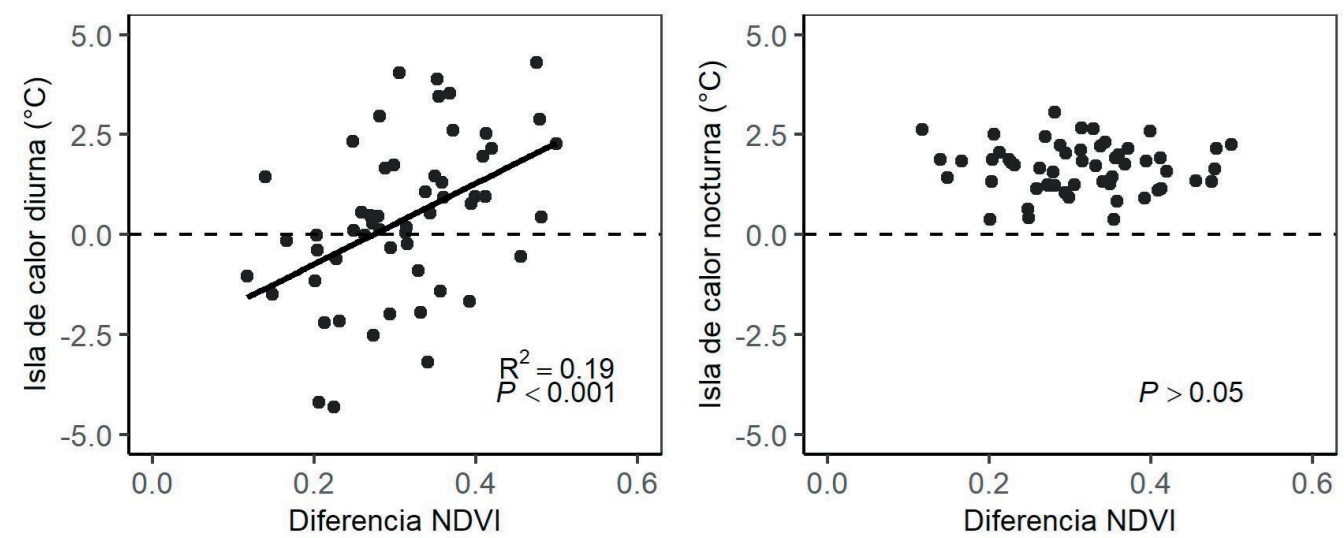

Figura 6. Isla de calor en relación con la diferencia entre NDVI no urbano y urbano durante (a) día y (b) noche. Cada punto representa la diferencia promedio por ciudad (2011-2015).

Figure 6. Urban heat island relationship with the NDVI difference between rural and urban areas. Each point represents the mean difference by city (2011-2015) (a) daytime and (b) nighttime.
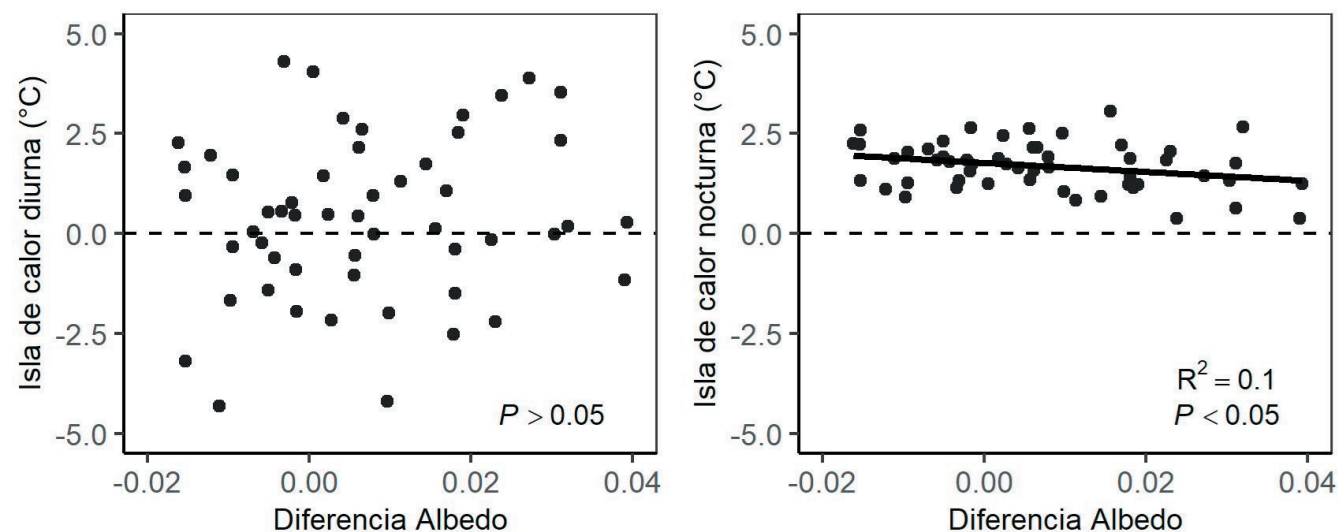

Figura 7. Islas de calor (a) diurna y (b) nocturna de las 55 ciudades en función de la diferencia de albedo azul entre las ciudades y su entorno (2011-2015).

Figure 7. Heat (a) diurnal and (b) nocturnal islands for the 55 cities according to the difference of blue albedo between cities and their non-urban surroundings (2011-2015). 
comportaron como IFU, aunque con gran variabilidad entre ciudades. La ICU nocturna fue independiente del lugar de emplazamiento de la ciudad. Las ciudades ubicadas en áreas con alto NDVI (i.e., Delta, Chaco Húmedo y Selva Paranaense) registran las mayores diferencias de TST diurna, siempre positivas durante el verano o primavera (Figura S5). En cambio, el invierno es la estación con menor intensidad de ICU, y fue el momento cuando ocurrieron las IFU de mayor magnitud (i.e., Monte, Espinal, Pampa). Las ciudades ubicadas en zonas más frías y secas (Monte) son las únicas ciudades que presentan siempre IFU diurnas.

\section{Discusión}

La ICU diurna promedio anual de las ciudades argentinas fue $0.36 \pm 1.99{ }^{\circ} \mathrm{C}$ y la nocturna fue $1.68 \pm 0.61{ }^{\circ} \mathrm{C}(n=55)$ (Figura 3$)$. La ICU nocturna siempre fue positiva y con menor variabilidad que la ICU diurna. Esta variabilidad es similar a la encontrada en 419 ciudades del mundo (Peng et al. 2012). En cambio, la ICU diurna promedio anual fue menor que el promedio global debido a la presencia de numerosas ciudades que se comportan como islas de frío. No se encontró correlación entre la ICU diurna y nocturna promedio anual a nivel regional (Figura S1), lo que sugiere controles diferentes durante el día y la noche. A nivel local, no se observó relación significativa entre valores diarios en diferentes ciudades (i.e., Río Cuarto, Neuquén, Corrientes) $(P>0.05)$ y podría indicar que existe poca inercia del proceso diurno sobre la noche siguiente (Peng et al. 2012).

\section{Patrones espaciales y controles de la ICU diurna}

La correlación entre la ICU diurna y la precipitación anual media (Figura 5b) sugiere que la ICU depende del clima local (Zhao et al. 2014). La relación es controlada por el cambio en la eficiencia convectiva asociada a cambios en la resistencia aerodinámica. En climas húmedos, la convección es menos eficiente disipando calor de las ciudades que de zonas rurales. La vegetación más densa genera más rugosidad y por lo tanto menor resistencia a la difusión de calor sensible. Lo contrario ocurre en regiones áridas, donde las zonas urbanas son más rugosas aumentando la eficiencia convectiva y el consecuente enfriamiento (Zhao et al. 2014).

Sin embargo, el rol de la vegetación como un control de la ICU diurna es evidente a lo largo del gradiente de vegetación en la Argentina. La correlación positiva de la ICU diurna con el NDVI no urbano $\left(R^{2}=0.51 ; P<0.001\right)$ (Figura S2a) evidencia la importancia del funcionamiento y estructura de la vegetación rural como referencia para indicar el impacto de la urbanización sobre la temperatura de superficie.

La intensidad de la ICU diurna promedio anual de las ciudades mostró un patrón espacial asociado al lugar de emplazamiento de las ciudades. En zonas dominadas por especies arbóreas y alto NDVI (e.g., ecorregiones Selva Paranaense y Yungas), las áreas urbanas están rodeadas de vegetación densa y alta. Esta vegetación intercepta, evapora y transpira la precipitación, manteniendo las regiones circundantes mucho más frías que en el núcleo urbano (diferencias de 1.7 a 4.5 ${ }^{\circ} \mathrm{C}$ ). Las áreas urbanas ubicadas en biomas dominados principalmente por especies herbáceas o menor proporción de leñosas y de baja biomasa - NDVI intermedio - como pastizales, arbustales y sabanas producen menor intensidad de ICU (-3.3 a $\left.2.9^{\circ} \mathrm{C}\right)$. En estas regiones, las zonas urbanas vegetadas restauran parte de la energía absorbida, reduciendo el gradiente horizontal de TST entre el centro de la ciudad y la zona rural (Imhoff et al. 2010). Así, el contraste de temperatura se debilita y hasta se revierte, como en la Región Pampeana. Las áreas urbanas rodeadas por desiertos y arbustos xéricos presentan un efecto de isla de frío con mayor frecuencia $\left(-4.3\right.$ a $\left.3.5^{\circ} \mathrm{C}\right)$. Este efecto de isla de frío fue observado en climas áridos y semiáridos, a pesar de altas densidades urbanas (Bounoua et al. 2009).

El efecto de la ICU se observó con mayor intensidad en biomas con alto NDVI como bosques o selvas que en pastizales o sabanas (Figura 4), pese al tamaño de las ciudades (las cuatro principales ciudades de la Argentina se encuentran en estos últimos biomas). El resultado coincide con investigaciones previas que mostraron que el impacto de la urbanización depende del bioma transformado y la intensidad de la modificación (Imhoff et al. 2010). Alterar relativamente poca área de una zona con mucha vegetación puede tener mayor impacto en el funcionamiento que modificaciones de grandes áreas con menor vegetación (Imhoff et al. 2010).

Es importante destacar la cantidad de islas de frío diurnas registradas (Figura 4). Las ciudades que registraron IFU anuales se 
ubican principalmente en el medio o extremo inferior del gradiente de NDVI no urbano (e.g., Espinal, Monte y Pampa). En general, las ciudades rodeadas por áreas rurales con suelos secos y desnudos (naturales o debido al uso del suelo) presentan condiciones favorables para la formación de islas de frío (Peña 2008) debido a la presencia de áreas vegetadas urbanas que generan enfriamiento por aumento de la evapotranspiración (Lazzarini et al. 2013). La vegetación del Espinal y el Monte se encuentra dominada por arbustos, con árboles dispersos y un estrato de gramíneas perennes (Duval et al. 2015). Estas formas de vida desarrollaron adaptaciones morfológicas, anatómicas y fisiológicas que reducen la superficie expuesta a la radiación o que disminuyen la transpiración (Duval et al. 2015) a partir de la apertura de estomas durante la noche, de generar tallos gruesos y espinas o de perder temporalmente las hojas (Grime 1977). En estas regiones, la actividad de la vegetación urbana podría ser mayor a su entorno. La mayor diversidad en los tipos de crecimiento dentro de las ciudades (e.g., perennifolias, caducifolias) y los subsidios de agua y nutrientes podrían contribuir al fenómeno. A pesar que el NDVI urbano sea siempre menor que la vegetación rural -debido a la menor proporción de superficie que la vegetación ocupa dentro del área urbana -, la vegetación urbana podría transpirar y crecer más activamente por el agua de riego (Pretzsch et al. 2017).

Otros factores pueden contribuir a la formación de IFU al reducir el impacto de la urbanización en la TST a pesar de tener poca superficie urbana vegetada (Imhoff et al. 2010). Ciertas propiedades de las urbanizaciones reducen la absorción de radiación solar al emplear materiales con alta reflectancia, baja conductividad térmica y alto albedo (Erell and Williamson 2007), además de que la presencia de construcciones altas puede generar sombra y reducir la energía que alcanza la superficie (Kato et al. 2010; Nassar et al. 2017).

Nuestros resultadosmuestranquevariaciones estacionales ICU de cada ciudad dependen del régimen climático y de la vegetación local. La intensidad de ICU es máxima durante el verano y menor en el invierno en ciudades cuya mayoría son templadas o subtropicales (Jenerette et al. 2011; Camilloni and Barrucand 2012), mientras que aquellas ciudades ubicadas en zonas tropicales exhiben una intensidad mayor en temporadas secas o frías (Roth 2007). Las ciudades ubicadas en regiones áridas o semiáridas mostraron islas de frío diurnas que se maximizan en el invierno (Figura S5). La amplitud entre estaciones se podría explicar parcialmente por el efecto de enfriamiento que produce la vegetación durante el período de crecimiento y el aumento de la transpiración (Peng et al. 2012; Imhoff et al. 2010). Por lo tanto, las ciudades con mayor contraste anual en la vegetación (NDVI) tienen mayor estacionalidad en la ICU, mientras que en aquellas ubicadas en áreas subtropicales donde la vegetación está activa todo el año la estacionalidad en la ICU es menor.

Muchas ciudades se comportan como ICU durante el verano e IFU en el invierno. Estas situaciones son coherentes con la mayor actividad fotosintética estival. Entre julio y octubre es posible encontrar el suelo en barbecho y más cálido que la vegetación activa aledaña (Buyantuyev and Wu 2010). La formación de islas de frío se debe a la menor inercia térmica de los suelos desnudos y secos en comparación con los materiales de construcción urbana (Peña 2008). La IFU en invierno en ciudades en zonas secas (Chaco Seco) es consistente con resultados de otros arbustales (Imhoff et al. 2010), a pesar de que la ICU media anual de las ciudades es positiva.

Laimportancia deluso de la tierra seevidencia al comparar ciudades con contextos ecológicos similares, pero ICU contrastantes. Neuquén y General Roca tienen ICU diurna, a diferencia de otras ciudades del Monte con IFU, debido a la mayor cobertura de vegetación herbácea y leñosa (frutales de carozo y pepita) que generan enfriamiento por evapotranspiración, a pesar del clima local semiárido. El NDVI no urbano aledaño a la ciudad podría determinar un comportamiento similar a las ciudades de zonas templadas, pero durante el invierno la vegetación de hojas caducas se asemeja al entorno de otras ciudades del Monte y genera islas de frío en toda la ecorregión.

\section{Patrones espaciales y controles de la ICU nocturna}

Los controles espaciales de la ICU nocturna no resultaron tan claros, ya que no se encontró relación con el clima ni con la vegetación natural o aledaña (Tabla S1). La baja evapotranspiración limita procesos de enfriamiento urbano y/o rurales. Por este motivo, la ICU nocturna fue relativamente estable a través de las estaciones al igual que en trabajos previos (Zhou et al. 2014; Yao et al. 2017). 
El almacenamiento de calor está relacionado con la absorción de energía solar durante el día, y las propiedades térmicas de la superficie, directamente asociadas con la urbanización. Es por ello que la caracterización del albedo y la extensión de la ciudad cobran relevancia. Todas las ciudades presentaron islas de calor nocturnas, independientemente del lugar de emplazamiento y el tamaño (superficie o población) debido al reemplazo de vegetación por superficie impermeable. Sin embargo, no se encontraron relaciones significativas entre la ICU nocturna y el tamaño como lo señala la bibliografía (Tabla S1; Oke 1973; Yao et al. 2017). Para eliminar efectos de otras variables se aislaron ciudades con contextos ecológicos similares, sin encontrar relaciones significativas.

El albedo es uno de los principales factores involucrados en la ICU nocturna (Peng et al. 2012; Zhou et al. 2014a). Las superficies impermeables tienen menor albedo, mayor conductividad térmica y capacidad de almacenar calor que las superficies rurales (Rizwan et al. 2008), por lo tanto, controlan positivamente a la isla de calor durante la noche. Nuestros resultados muestran una correlación negativa entre la ICU nocturna anual y la diferencia de albedo entre áreas urbanas y no urbanas $\left(R^{2}=0.1, P<0.05\right)$. La pendiente de la relación indica que zonas con mayor diferencia positiva entre el albedo no urbano y el urbano generan menor isla de calor nocturna. Estos resultados sugieren el rol del almacenamiento del calor para controlar la ICU nocturna (Peng et al. 2012). Investigaciones futuras deberían evaluar la importancia de la emisión de calor antropogénico por actividades socioeconómicas, por ejemplo, datos provistos por sensores remotos de luz nocturna (Peng et al. 2012).

\section{Influencia de vegetación urbana}

La vegetación urbana tiene importancia en la regulación de la temperatura tanto por su rol directo al transpirar agua como por su relación inversa con la superficie impermeabilizada. Mayores diferencias de NDVI se correlacionaron con mayores cambios en la TST diurna en el área urbana con respecto al entorno independientemente del contexto ecológico (Figura 6a). Sin embargo, a pesar de las evidencias que indican la correlación negativa entre el NDVI urbano y la TST urbana diurna (Buyantuyev and Wu 2010; Shi and Zhang 2017), no observó esta correlación en el análisis regional de las ICU de la Argentina.
Este efecto pudo haberse enmascarado debido a la influencia de otros factores como extensión o contexto de las ciudades. El análisis de la relación entre ICU y NDVI urbano para las ciudades de cada ecorregión sólo mostró relación negativa para Chaco Seco y una relación positiva en la región Pampeana (Tabla S3).

La ICU nocturna y el NDVI urbano se correlacionaron negativamente. La ausencia de enfriamiento por evapotranspiración de la vegetación disminuye la influencia sobre la intensidad de la isla de calor. A pesar de que se sugiere que la vegetación no modifica la ICU nocturna (Zhou et al. 2014b; Zhou et al. 2016), nuestros resultados concuerdan con los de otros estudios (Yao et al. 2017). La correlación negativa puede indicar que una vegetación más densa y activa en áreas urbanas aumentaría las zonas de sombra y disminuiría las superficies impermeables, y de esta manera reduciría el almacenamiento de calor diurno que será liberado durante la noche.

\section{Incertidumbre y sugerencias}

La definición de isla de calor utilizada en este trabajo y en muchos otros trabajos publicados depende del área no urbana utilizada como referencia. Por lo tanto, áreas urbanas con la misma temperatura, pero con usos de la tierra no urbanos en la periferia disímiles, pueden determinar en gran medida la magnitud y el sentido de la ICU. Los cambios en el uso de la tierra alrededor de las ciudades (e.g., mayor cobertura de cultivos de verano frente invierno, menor cobertura de doble cultivo trigo/soja) afectan claramente los resultados presentados e implican la necesidad de buscar alternativas metodológicas. Otros indicadores de ICU derivados con teledetección a considerar en un futuro son la desviación estándar de TST en una ciudad (Schwarz et al. 2011) o la altura de una campana de Gauss con el aumento de TST en relación con la superficie (Hung et al. 2006; Streutker 2002).

Este análisis regional en la Argentina evidencia que el contexto de emplazamiento del área urbana resulta más importante por sobre el tipo de urbanización. Para una efectiva mitigación del fenómeno ICU y el planeamiento territorial se deberían tomar decisiones locales para desarrollar ciudades mejor diseñadas e intervenidas que podrían ser más eficientes en términos energéticos. Frente a la tendencia de aumento de la población 
urbana será importante asegurar superficies que amortigüen la temperatura como cuerpos de agua (Hathway and Sharples 2012) o espacios verdes (tanto públicos, privados $\mathrm{u}$ otros como los techos verdes) (Feyisa et al. 2014), así como disminuir superficies impermeables (Imhoff et al. 2010).

\section{Conclusiones}

El contexto ecológico fue el principal modulador de la variación espacio-temporal de las temperaturas de superficie y la generación de islas de calor e islas de frío. Ciudades similares en regiones más húmedas y verdes (superficie y valor de NDVI) presentaron las mayores diferencias de temperatura con el entorno debido principalmente al cambio de evapotranspiración por reemplazo de cobertura y la disminución en la eficiencia para disipar calor. Las ciudades ubicadas en sitios más secos con NDVI más bajo tuvieron ICU de menor intensidad o inversión térmica (i.e., islas de frío).

Mayores diferencias entre NDVI urbano y no urbano correlacionaron con mayores diferencias de temperatura, lo cual indica la influencia de la vegetación en la ICU independientemente del contexto. Sin embargo, no se pudo comprobar la influencia de espacios verdes urbanos en el enfriamiento de las ciudades a nivel regional. Por un lado, las ciudades con mayor NDVI urbano tuvieron mayor ICU. Por otro lado, en contextos ecológicos similares, una ciudad con mayor NDVI, por cobertura vegetada o densidad de espacios verdes, no necesariamente tendrá mayor ICU que otra con menor vegetación urbana. La ICU nocturna fue siempre positiva y con baja variabilidad estacional. El aumento de la temperatura nocturna resultó independiente de contextos ambientales pero ligados a la estructura urbana (diferencia de albedo y NDVI urbano).

Este trabajo representa la primera descripción del impacto de la urbanización sobre los climas urbanos en un gradiente regional en la Argentina. Pone en evidencia la importancia tanto de las características de la ciudad (i.e., superficie, vegetación urbana, albedo) como también de las zonas no urbanas. Nuestros resultados proveen una mejor comprensión de la magnitud y la extensión espacial de las islas de calor urbano y de algunos de sus controles principales.

Agradecimientos. A M. Oesterheld por la gran ayuda con herramientas clave para el trabajo, a T. Propato y S. Verón por las discusiones que enriquecieron el análisis realizado, y a D. Villarroel Torrez por su colaboración en diferentes etapas del análisis. Este trabajo fue financiado por la Universidad de Buenos Aires (programación UBACyT 2016).

\section{REFERENCIAS}

Angel, S., J. Parent, D. L. Civco, A. Blei, and D. Potere. 2011. The dimensions of global urban expansion: Estimates and projections for all countries, 2000-2050. Progress in Planning 75:53-107. https://doi.org/10.1016/ j.progress.2011.04.001.

Bonet, J., S. Levy, M. Marcel, and A. M. Rosa. 2011. Sostenibilidad urbana en América Latina y el Caribe. Banco Interamericano de Desarrollo. 3-8. Acceso en noviembre 2015. URL: tinyurl.com/yc59kuv9.

Bounoua, L., A. Safia, J. Masek, C. Peters-Lidard, and M. L. Imhoff. 2009. Impact of urban growth on surface climate: A case study in Oran, Algeria. Journal of Applied Meteorology and Climatology. 48:217-231. https://doi.org/10.1175/ 2008JAMC2044.1.

Buyantuyev, A., and J. Wu. 2010. Urban heat islands and landscape heterogeneity: linking spatiotemporal variations in surface temperatures to land-cover and socioeconomic patterns. Landscape Ecology 251:17-33. https://doi.org/ 10.1007/s10980-009-9402-4.

Correa, E., C. Rosa, and G. Lesino. 2006. Monitoreo de Clima Urbano: análisis estadístico de los factores que determinan la isla de calor y su aporte al diseño de los espacios urbanos. AVERMA Avances en Energías Renovables y Medio Ambiente 10:41-48.

de la Casa, A. C., and O. B. Nasello. 2014. Efectos del calentamiento global sobre el consumo de energía en la ciudad de Córdoba, Argentina. ANALES AFA 25:18-23. https://doi.org/10.31527/analesafa.2014.25.1.18.

Duval, V. S., G. M. Benedetti, and A. M. Campo. 2015. Relación clima-vegetación: adaptaciones de la comunidad del jarillal al clima semiárido, Parque Nacional Lihué Calel, provincia de La Pampa, Argentina. Investigaciones Geográficas 88:33-44. https://doi.org/10.14350/rig.48033.

Erell, E., and T. Williamson. 2007. Intra-urban differences in canopy layer air temperature at a mid-latitude city. International Journal of Climatology 27:1243-1255. https://doi.org/10.1002/joc.1469.

Farr, T. G., P. A. Rosen, E. Caro, R. Crippen, R. Duren, S. Hensley, M. Kobrick, M. Paller, E. Rodríguez, L. Roth, D. Seal, S. Shaffer, J. Shimada, J. Umland, M. Werner, M. Oskin, D. Burbank, and D. E. Alsdorf. 2007. The shuttle radar topography mission. Reviews of Geophysics 45:RG2004. https://doi.org/10.1029/2005RG000183.

Friedl, M. A., D. Sulla-Menashe, B. Tan, A. Schneider, N. Ramankutty, A. Sibley, and X. Huang. 2010. MODIS Collection 
5 global land cover: Algorithm refinements and characterization of new datasets. Remote Sensing of Environment 114:168-182. https://doi.org/10.1016/j.rse.2009.08.016.

Garbulsky, M. F., J. Peñuelas, J. Gamon, Y. Inoue, and I. Filella. 2011. The photochemical reflectance index (PRI) and the remote sensing of leaf, canopy and ecosystem radiation use efficiencies. A review and meta-analysis. Remote Sensing of Environment 115:281-297. https://doi.org/10.1016/j.rse.2010.08.023.

Garbulsky, M. F., I. Filella, A. Verger, and J. Peñuelas. 2014. Photosynthetic light use efficiency from satellite sensors: from global to Mediterranean vegetation. Environmental and Experimental Botany 103:3-11. https://doi.org/10.1016/ j.envexpbot.2013.10.009.

Gioia, A., L. Paolini, A. Malizia, R. Oltra-Carrió, and J. A. Sobrino. 2014. Size matters: vegetation patch size and surface temperature relationship in foothills cities of northwestern Argentina. Urban Ecosystems 17:1161-1174. https: //doi.org/10.1007/s11252-014-0372-1.

Grimm, N. B., D. Foster, P. Groffman, J. M. Grove, C. S. Hopkinson, K. J. Nadelhoffer, and D. P. Peters. 2008. The changing landscape: ecosystem responses to urbanization and pollution across climatic and societal gradients. Frontiers in Ecology and the Environment 6:264-272. https://doi.org/10.1890/070147.

Groffman, P. M., J. Cavender-Bares, N. D. Bettez, J. M. Grove, S. J. Hall, J. B. Heffernan, and K. Nelson. 2014. Ecological homogenization of urban USA. Frontiers in Ecology and the Environment 12:74-81. https://doi.org/10.1890/120374.

Hathway, E. A., and S. Sharples. 2012. The interaction of rivers and urban from in mitigating the urban heat island effect: a UK case study. Building and Environment 58:14-22. https://doi.org/10.1016/j.buildenv.2012.06.013.

Hung, T., D. Uchihama, S. Ochi, and Y. Yasuoka. 2006. Assessment with satellite data of the urban heat island effects in Asian mega cities. International Journal of Applied Earth Observation and Geoinformation 8:34-48. https://doi.org/ 10.1016/j.jag.2005.05.003.

Imhoff, M. L., P. Zhang, R. E. Wolfe, and L. Bounoua. 2010. Remote sensing of the urban heat island effect across biomes in the continental USA. Remote Sensing of Environment 114:504-513. https://doi.org/10.1016/j.rse.2009.10.008.

INDEC (Instituto Nacional de Estadística y Censos). 2010. Encuesta Permanente de Hogares - EPH Continua [Base de datos en línea], Acceso online diciembre 2020. URL: tinyurl.com/INDECcenso2010.

IGN, Instituto Geográfico Nacional de la República Argentina. 2017. Planta urbana. Acceso online diciembre 2019. URL: tinyurl.com/CapasSIG.

Jin, M., R. E. Dickinson, and D. A. Zhang. 2005. The footprint of urban areas on global climate as characterized by MODIS. Journal of Climate 18:1551-1565. https://doi.org/10.1175/JCLI3334.1.

Jenerette, G. D., S. L. Harlan, W. L. Stefanov, and C. A. Martin. 2011. Ecosystem services and urban heat riskscape moderation: water, green spaces, and social inequality in Phoenix, USA. Ecological Applications 21:2637-2651. https: //doi.org/10.1890/10-1493.1.

Kato, S., T. Matsunaga, and Y. Yamaguchi. 2010. Influence of Shade on Surface Temperature in an Urban Area Estimated by ASTER Data. International Archives of the Photogammetry, Remote Sensing and Spatial Information Science, Kyoto Japan 38 (part 8).

Lazzarini, M., P. R. Marpu, and H. Ghedira. 2013. Temperature-land cover interactions: the inversion of urban heat island phenomenon in desert city areas. Remote Sensing of Environment 130:136-152. https://doi.org/10.1016/ j.rse.2012.11.007.

Maristany, A., L. Abadía, S. Angiolini, A. Pacharoni, and M. Pardina. 2008. Estudio del fenómeno de la isla de calor en la ciudad de Córdoba-Resultados preliminares. Avances en Energías Renovables y Medio Ambiente 12:11-69.

McKinney, M. L. 2006. Urbanization as a major cause of biotic homogenization. Biological Conservation 127:247-260. https://doi.org/10.1016/j.biocon.2005.09.005.

Mills, G. 2007. Cities as agents of global change. International Journal of Climatology 27:1849-1857. https://doi.org/ 10.1002/joc. 1604 .

Naciones Unidas, Departamento de Asuntos Económicos y Sociales, División de Población 2018. World Urbanization Prospects: The 2018 Revision, Nueva York, Naciones Unidas. Último acceso: diciembre de 2018. URL: population.un.org/ wup.

Nassar, A. K., G. A. Blackburn, and J. D. Whyatt. 2017. What controls the magnitude of the daytime heat sink in a desert city? Applied Geography 80:1-14. https://doi.org/10.1016/j.apgeog.2017.01.003.

Oke, T. R. 1973. City size and the urban heat island. Atmospheric Environment 7:769-779. https://doi.org/10.1016/00046981(73)90140-6.

Olson, D. M., E. Dinerstein, E. Wikramanayake, N. D. Burgess, G. Powell, E. C. Underwood, J. A. D’amico, I. Itoua, H. E. Strand, J. C. Morrison, C. J. Loucks, T. F. Allnutt, T. H. Ricketts, Y. Kura, J. F. Lamoreux, W. W. Wettengel, P. Hedao, and K. R. Kassem. 2001. Terrestrial Ecoregions of the World: A New Map of Life on Earth A new global map of terrestrial ecoregions provides an innovative tool for conserving biodiversity. BioScience 51:933-938. https: //doi.org/10.1641/0006-3568(2001)051[0933:TEOTWA]2.0.CO;2.

Paolini, L. 2012. Análisis de la respuesta de la temperatura de superficie al crecimiento urbano utilizando series temporales MODIS. Revista de Teledetección 38:19-26.

Peng, S., S. Piao, P. Ciais, P. Friedlingstein, C. Ottle, F. M. Breìn, and R. B. Myneni. 2012. Surface urban heat island across 419 global big cities. Environmental Science and Technology 46:696-703. https://doi.org/10.1021/es2030438.

Peña, M. A. 2008. Relationships between remotely sensed surface parameters associated with the urban heat sink formation in Santiago, Chile. International Journal of Remote Sensing 29:4385-4404. https://doi.org/10.1080/ 01431160801908137. 
Pettorelli, N., J. O. Vik, A. Mysterud, J. M. Gaillard, C. J. Tucker, and N. C. Stenseth. 2005. Using the satellite-derived NDVI to assess ecological responses to environmental change. Trends in Ecology and Evolution 20:503-510. https: //doi.org/10.1016/j.tree.2005.05.011.

Porteous, A. 2013. Dictionary of environmental science and technology. Fourth edition. John Wiley and Sons, Chichester, West Sussex, England.

Pretzsch, H., P. Biber, E. Uhl, J. Dahlhausen, G. Schütze, D. Perkins, T. Rötzer, J. Caldentey, T. Koike, T. van Con, A. Chavanne, B. du Toit, K. Foster, and Lefer B. 2017. Climate change accelerates growth of urban trees in metropolises worldwide. Scientific Reports 7:15403. https://doi.org/10.1038/s41598-017-14831-w.

Rizwan, A. M., L. Y. Dennis, and L. I. U. Chunho. 2008. A review on the generation, determination and mitigation of Urban Heat Island. Journal of Environmental Sciences 20:120-128. https://doi.org/10.1016/S1001-0742(08)60019-4.

Roth, M. 2007. Review of urban climate research in (sub) tropical regions. International Journal of Climatology 27: 1859-1873. https://doi.org/10.1002/joc.1591.

Roth, M., T. R. Oke, and W. J. Emery. 1989. Satellite-derived urban heat islands from three coastal cities and the utilization of such data in urban climatology. International Journal of Remote Sensing 10:1699-1720. https://doi.org/ 10.1080/01431168908904002.

Schwarz, N., S. Lautenbach, and R. Seppelt. 2011. Exploring indicators for quantifying surface urban heat islands of European cities with MODIS land surface temperatures. Remote Sensing of Environment 115:3175-3186. https: //doi.org/10.1016/j.rse.2011.07.003.

Shi, Y., and Y. Zhang. 2017. Remote sensing retrieval of urban land surface temperature in hot-humid region. Urban Climate 24:299-310. https://doi.org/10.1016/j.uclim.2017.01.001.

Streutker, D. R. 2002. A remote sensing study of the urban heat island of Houston, Texas. International Journal of Remote Sensing 23:2595-2608. https://doi.org/10.1080/01431160110115023.

Taheri Shahraiyni, H., S. Sodoudi, A. El-Zafarany, T. Abou El Seoud, H. Ashraf, and K. Krone. 2016. A Comprehensive Statistical Study on Daytime Surface Urban Heat Island during summer in Urban Areas, Case Study: Cairo and Its New Towns. Remote Sensing 8:643. https://doi.org/10.3390/rs8080643.

Verón, E. M. 2010. Estimación de la isla de calor en Santa Teresita, Partido de la Costa, Provincia de Buenos Aires, Argentina. Revista Geográfica de América Central 45:129-148.

Walker, J. J., K. M. de Beurs, and G. M. Henebry. 2015. Land surface phenology along urban to rural gradients in the US Great Plains. Remote Sensing of Environment 165:42-52. https://doi.org/10.1016/j.rse.2015.04.019.

Wan, Z. 2006. MODIS land surface temperature products users' guide. Institute for Computational Earth System Science, University of California, Santa Barbara, USA.

Wan, Z., S. Hook, G. Hulley. 2015. MYD11A2 MODIS/Aqua Land Surface Temperature/Emissivity 8-Day L3 Global $1 \mathrm{~km}$ SIN Grid V006 [Data set]. NASA EOSDIS Land Processes DAAC. Último acceso: 3 de marzo 2021: $\underline{\mathrm{https}} / / \mathrm{doi}$. org/ 10.5067/MODIS/MYD11A2.006.

Wong, K., A. Paddon, and A. Jimenez. 2013. Review of world urban heat islands: Many linked to increased mortality. Journal of Energy Resources Technology 135:1-11. https://doi.org/10.1115/1.4023176.

Yao, R., L. Wang, X. Huang, Z. Niu, F. Liu, and Q. Wang. 2017. Temporal trends of surface urban heat islands and associated determinants in major Chinese cities. Science of the Total Environment 609:742-754. https://doi.org/10.1016/ j.scitotenv.2017.07.217.

Zhao, L., X. Lee, R. B. Smith, and K. Oleson. 2014. Strong contributions of local background climate to urban heat islands. Nature 511:216-219. https://doi.org/10.1038/nature13462.

Zhou, W., Y. Qian, X. Li, W. Li, and L. Han. 2014a. Relationships between land cover and the surface urban heat island: seasonal variability and effects of spatial and thematic resolution of land cover data on predicting land surface temperatures. Landscape Ecology 29:153-167. https://doi.org/10.1007/s10980-013-9950-5.

Zhou, D., S. Zhao, S. Liu, L. Zhang, and C. Zhu. 2014b. Surface urban heat island in China's 32 major cities: spatial patterns and drivers. Remote Sensing Environment 152:51-61. https://doi.org/10.1016/j.rse.2014.05.017.

Zhou, D., L. Zhang, D. Li, D. Huang, and C. Zhu. 2016. Climate-vegetation control on the diurnal and seasonal variations of surface urban heat islands in China. Environmental Research Letters 11:074009. https://doi.org/10.1088/ 1748-9326/11/7/074009.

Zhou, B., D. Rybski, and J. P. Kropp. 2017. The role of city size and urban form in the surface urban heat island. Scientific Reports 7:4791. https://doi.org/10.1038/s41598-017-04242-2. 